\title{
Intake of ruminant trans-fatty acids, assessed by diet history interview, and changes in measured body size, shape and composition
}

\author{
Camilla P Hansen $1,2,3, *$, Berit L Heitmann ${ }^{4,5,6}$, Thorkild IA Sørensen ${ }^{3,7}$, Kim Overvad ${ }^{1,2}$ \\ and Marianne U Jakobsen ${ }^{1}$ \\ 'Section for Epidemiology, Department of Public Health, Aarhus University, Bartholins Allé 2, 8000 Aarhus C, \\ Denmark: ${ }^{2}$ Department of Cardiology, Center for Cardiovascular Research, Aalborg University Hospital, \\ Aalborg, Denmark: ${ }^{3}$ Institute of Preventive Medicine, Bispebjerg and Frederiksberg Hospital, The Capital Region, \\ Copenhagen, Denmark: ${ }^{4}$ Research Unit for Dietary Studies, The Parker Institute, Bispebjerg and Frederiksberg \\ Hospital, The Capital Region, Copenhagen, Denmark: ${ }^{5}$ Research Centre for Prevention and Health, Glostrup \\ Hospital, Glostrup, Denmark: ${ }^{6}$ National Institute of Public Health, University of Southern Denmark, Copenhagen, \\ Denmark: ${ }^{7}$ Novo Nordisk Foundation Center for Basic Metabolic Research, University of Copenhagen, \\ Copenhagen, Denmark
}

Submitted 19 August 2014: Final revision received 21 March 2015: Accepted 2 May 2015: First published online 15 June 2015

\begin{abstract}
Objective: Studies have suggested that total intake of trans-fatty acids (TFA) is positively associated with changes in body weight and waist circumference, whereas intake of TFA from ruminant dairy and meat products (R-TFA) has not been associated with weight gain. However, these previous studies are limited by self-reported measures of body weight and waist circumference or by a crosssectional design. The objective of the present study was to investigate if R-TFA intake was associated with subsequent changes in anthropometry (body weight, waist and hip circumference) measured by technicians and body composition (body fat percentage).

Design: A 6-year follow-up study. Information on dietary intake was collected through diet history interviews, and anthropometric and bioelectrical impedance measurements were obtained by trained technicians at baseline (1987-1988) and at follow-up (1993-1994). Multiple regression with cubic spline modelling was used to analyse the data.

Setting: Copenhagen County, Denmark.

Subjects: Two hundred and sixty-seven men and women aged 35-65 years from the Danish MONICA (MONItoring of trends and determinants in CArdiovascular diseases) cohort.

Results: The median R-TFA intake was $1.3 \mathrm{~g} / \mathrm{d}$ ( 5 th, 95 th percentile: $0 \cdot 4,2 \cdot 7 \mathrm{~g} / \mathrm{d}$ ) or $0 \cdot 6 \%$ of the total energy intake ( 5 th, 95 th percentile: $0 \cdot 2,1 \cdot 1 \%$ ). No significant associations were observed between R-TFA intake and changes in body weight, waist and hip circumference or body fat percentage.

Conclusions: R-TFA intake within the range present in the Danish population was not significantly associated with subsequent changes in body size, shape or composition and the $95 \%$ confidence intervals indicate that any relevant associations are unlikely to have produced these observations.
\end{abstract}

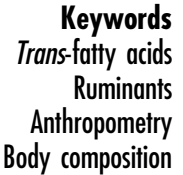

Trans-fatty acids (TFA) are unsaturated fatty acids with at least one double bond in trans configuration. TFA in the diet originate from ruminant dairy and meat products and from margarines and shortenings containing industrial partially hydrogenated oils. A special group of TFA is conjugated linoleic acids (CLA), which have both a cis and a trans double bond separated by two $\mathrm{CH}_{2}$ groups.
Rumenic acid (cis 9, trans 11-18:2 (18:2 $\Delta 9 c, 11 t))$, a CLA isomer, is present in ruminant dairy and meat products. In addition, vaccenic acid (trans 11-18: $1(18: 1 \Delta 11 t)$ ), the main isomer among TFA from ruminant sources (R-TFA), can be converted to rumenic acid endogenously in $\operatorname{man}^{(1,2)}$. Animal studies have found that feeding CLA reduces body weight and fat deposition; the results from 
human studies are, however, inconsistent ${ }^{(3,4)}$. The content of TFA in margarines and shortenings available on the Danish market has been reduced, and as a result the average intake of TFA from these sources decreased from $2 \cdot 2 \mathrm{~g} / \mathrm{d}$ in 1992 to $0.4 \mathrm{~g} / \mathrm{d}$ in $1999^{(5)}$. In 2004, Denmark further imposed a restriction on the content of industrially produced TFA (I-TFA) in all margarines and shortenings, and thus I-TFA no longer contribute notably to the total intake of TFA ${ }^{(6)}$. The median intake of R-TFA in the Danish population aged 1-80 years has been estimated as $1.7 \mathrm{~g} / \mathrm{d}$ ( $0.7 \%$ of energy intake) with the $80 \%$ central range being $0.9-2.7 \mathrm{~g} / \mathrm{d}(0.5-1.0 \%$ of energy intake); dairy products being the main source ${ }^{(7)}$. The consumption of I-TFA is also decreasing worldwide ${ }^{(8,9)}$. However, it has been estimated that even if partially hydrogenated oils were eliminated from the food supply, TFA would still contribute approximately $1 \%$ of energy intake in the USA due to the natural occurrence of TFA in dairy and meat products ${ }^{(10)}$.

Previous studies have suggested that TFA may play a role in the development of obesity ${ }^{(11)}$. Two large American cohort studies found positive associations between total intake of TFA and changes in weight and waist circumference $^{(12,13)}$. In contrast, we previously observed a weak inverse association between R-TFA intake and weight change, whereas no substantial association with change in waist circumference was found ${ }^{(14)}$. Two crosssectional studies found no significant associations between R-TFA and BMI or waist circumference ${ }^{(15,16)}$. In a cross-sectional study of adipose tissue TFA, rumenic acid was positively associated with skinfold thickness, but not with BMI or waist circumference, whereas trans 9-16:1 (16:1 $\Delta 9 t)$, which is also derived from ruminant food sources, was likewise not associated with BMI or waist circumference, but inversely associated with skinfold thickness ${ }^{(17)}$. However, these previous studies have limitations as weight and waist circumference measurements were self-reported, dietary data were collected with FFQ or the study design was cross-sectional ${ }^{(14-17)}$.

The aim of the present study was therefore to investigate the associations between intake of R-TFA, assessed by a diet history interview, and subsequent 6-year changes in body size and shape, measured by trained technicians, and body composition obtained by bioelectrical impedance analysis.

\section{Methods}

\section{Study population and design}

The study population comprised 552 men and women aged 35, 45, 55 and 65 years, selected randomly from a larger sex- and age-stratified sample of the population in the western part of Copenhagen County, which was part of the follow-up sample in the Danish MONICA (MONItoring of trends and determinants in CArdiovascular diseases) cohort. In 1982-1983, 4807 Danish citizens born in 1922, 1932, 1942 or 1952 were invited to participate in the MONICA-I survey. After excluding persons not born in Denmark, the sample was reduced to 4581 individuals of whom 3608 (79\%) participated. The participants were later invited to two re-examinations. The first re-examination (the GEN-MONICA survey) was carried out from December 1987 to November 1988, where all participants were invited to a general health examination including anthropometric and bioelectrical impedance measurements and a random sub-sample ( $n$ 552) was invited to give diet history interviews in addition to the health examination. Information on anthropometric and bioelectrical impedance measurements was also collected at the second re-examination (the MONICA10 survey) carried out from June 1993 to September 1994. In the present study, the GEN-MONICA survey constitutes the baseline and the MONICA10 survey constitutes the followup. This study was conducted according to the guidelines laid down in the Declaration of Helsinki and all procedures involving human subjects were approved by the ethics committee for Copenhagen County. Written informed consent was obtained from all participants.

\section{Dietary data}

Diet history interviews conducted by the same trained dietitian at the day of the health examination were used to collect information on the habitual diet of the participants. Meal patterns, dishes and foods during the previous month were explored asking non-leading open-ended questions, while quantities were assessed by food models, photograph series and household measures. Nutrient calculations were done using a computer program (DANKOST) based on the Danish food composition tables ${ }^{(18)}$. R-TFA intake was estimated by combining information on food intake with the content of TFA in milk fat ${ }^{(7)}$ and ruminant meat products ${ }^{(19)}$.

A weighted intake of foods containing high amounts of I-TFA was calculated by selecting and weighting food items according to estimates of the content of I-TFA in Danish foods. It was assumed that the weighted intake would rank the participants according to I-TFA intake, in spite of the fact that the content of I-TFA in margarines and foods has changed since the dietary information was obtained $^{(20)}$.

\section{Anthropometric measurements and bioelectrical impedance analysis}

Anthropometric measurements both at baseline and at follow-up were made by trained technicians in accordance with the WHO standards ${ }^{(21)}$. Height was measured for participants standing without shoes, heels together and head in the Frankfurt horizontal plane and recorded to the nearest $0.5 \mathrm{~cm}$. Body weight was measured with a SECA scale with participants wearing underwear or light clothes and recorded to the nearest $0 \cdot 1 \mathrm{~kg}$. Waist circumference 
was measured halfway between the lower rib and the iliac crest, and hip circumference was measured at the point yielding the maximum circumference over the buttocks. Both circumference measurements were recorded to the nearest $0.5 \mathrm{~cm}$.

Electrical impedance was measured with a BIA-103 analyser (RJL Systems, Detroit, MI, USA) using a tetrapolar electrode placement. The measurement was made with participants relaxed in supine position and electrodes placed on the dorsal surfaces of the right hand and foot, at the distal metacarpals and metatarsals, and between the distal prominences of the radius and the ulna at the wrist and the medial and lateral malleoli at the ankle. The following algorithm, previously developed based on a subgroup of the present random sample of Danes ${ }^{\text {(21) }}$, was used to estimate body fat from electrical impedance:

$$
\begin{aligned}
\mathrm{BF}= & 0 \cdot 819 \mathrm{BW}-0 \cdot 279 \times \mathrm{H}^{2} / \mathrm{R}-\mathrm{sex} \times \mathrm{BW}+0 \cdot 77 \mathrm{age} \\
& -0 \cdot 231 \mathrm{H}+14 \cdot 941,
\end{aligned}
$$

where $\mathrm{BF}=$ body fat $(\mathrm{kg}), \mathrm{BW}=$ body weight $(\mathrm{kg})$, $\mathrm{H}=$ height $(\mathrm{cm}), \mathrm{R}=$ resistance $(\Omega)$ and $\mathrm{sex}=1$ for men and 0 for women. Fatness (body fat percentage) was calculated as $\mathrm{BF} / \mathrm{BW} \times 100$.

The outcomes in the present study were defined as average annual changes in body weight, waist and hip circumference and body fat percentage, calculated by subtracting the baseline values from the follow-up values and dividing these differences by the duration of follow-up time.

\section{Other covariates}

An extensive questionnaire on lifestyle and sociodemographic factors was sent to the participants before the health examination. Leisure-time physical activity was recorded in four categories based on questions originally constructed and evaluated by Saltin and Grimby ${ }^{(22)}$ : (i) mostly sedentary; (ii) walking, bicycling or other light physical activity for at least $4 \mathrm{~h} /$ week; (iii) participating in sports for at least $3 \mathrm{~h} /$ week; and (iv) competitive sports or long-distance running several times per week. The last two categories were merged into one due to scanty data in the last category. The participants recorded their smoking habits as daily smoker, occasional smoker (smoking less than one cigarette daily), former smoker or non-smoker. Based on the information about smoking at baseline and follow-up, a new variable was generated classifying the participants as having no changes in smoking habits, initiating daily smoking or quitting daily smoking. Education was recorded as number of years in school and subsequently categorised as $\leq 7$ years, 8-11 years and $\geq 12$ years.

\section{Exclusion criteria}

Participants were excluded if data on intake of R-TFA, weight, waist circumference, hip circumference, bioelectrical impedance analysis (not obtained in pregnant women), follow-up time or any of the potential confounders were missing. Further, it was decided to exclude participants with any of the following chronic diseases at baseline or follow-up: cancer (ICD-7 codes: 140-148, 150-165, 170-181 and 192-207), CVD (ICD-8 codes: 390-458 and ICD-10 code: I00-I99) and diabetes (selfreported) as these diseases and their treatment might cause changes in diet and anthropometry (ICD = International Classification of Diseases).

\section{Statistical analyses}

The associations between intake of R-TFA and average annual change in anthropometry (body weight, waist and hip circumference) and body composition (body fat percentage) were investigated using multiple linear regression analysis.

Two different modelling approaches were used. First, R-TFA was included in $\mathrm{g} / \mathrm{d}$ without adjustment for total energy intake and other important determinants of variation in energy balance between persons (i.e. physical activity). Second, R-TFA was included as a nutrient density (energy intake from R-TFA divided by total energy intake) together with total energy intake (including energy intake from alcohol) and leisure-time physical activity (mostly sedentary; walking, bicycling or other light physical activity for at least $4 \mathrm{~h} /$ week; participating in sports for at least $3 \mathrm{~h} /$ week $^{(23)}$. To control for confounding, the following variables were included as covariates in the models : sex, age (35 years, 45 years, 55 years and 65 years), baseline BMI, baseline waist circumference (only in the analyses of changes in waist circumference), baseline hip circumference (only in the analyses of changes in hip circumference), baseline body fat percentage (only in the analyses of changes in fatness), smoking habits (constant, initiate smoking, quit smoking), weighted intake of foods containing high amounts of I-TFA $(\mathrm{g} / \mathrm{d})$ and education ( $\leq 7$ years, $8-11$ years and $\geq 12$ years). Ca and protein intakes were also adjusted for in sub-analyses as studies have suggested that Ca intake ${ }^{(24-26)}$ and protein intake ${ }^{(27,28)}$ may protect against weight gain.

All continuous covariates were included as restricted cubic splines with three knots at the 10th, 50th and 90th percentile ${ }^{(29,30)}$. Intake of R-TFA was modelled using cubic splines as in another cohort we observed non-linear associations between R-TFA intake and changes in weight and waist circumference ${ }^{(14)}$. Associations were considered statistically significant if at least one of the two spline parameters differed significantly from zero as assessed by the Wald test. The associations between R-TFA intake and changes in anthropometry and body composition were considered to be non-linear if the last spline parameter differed significantly from zero as assessed by the Wald test. If the test for non-linearity was not statistically significant, linear regression coefficients for the associations between R-TFA intake and changes in anthropometry and body composition were calculated. The interaction 
between R-TFA and sex was tested by adding a product term to the model. Two-sided $P$ values below 0.05 were considered statistically significant.

Residual- $v$.-fitted plots were used to check the assumption of homogeneity of variance, while histograms and $\mathrm{P}-\mathrm{P}$ plots of the residuals were used to check the assumption of normally distributed error terms. No violations were found, except that some signs of heterogeneity of variances between men and women were observed in the analyses of changes in hip circumference. Therefore, results from regression analyses conducted with robust standard errors (Huber/White) are reported for changes in hip circumference.

Data were analysed using the Stata statistical software package version $12 \cdot 1$.

\section{Results}

Of the 552 individuals invited to complete a diet history interview in GEN-MONICA, 434 (79\%) accepted; $76 \%$ ( $n$ 329) of those with a complete diet history interview also participated in the MONICA10 follow-up and were thus eligible for inclusion in the present study. Information on waist circumference was missing for one individual and bioelectrical impedance measures were missing in seven. In addition, two individuals were excluded due to missing information on leisure-time physical activity. Finally, participants with prevalent disease or developing disease during follow-up were excluded: twelve persons with cancer, thirty-four persons with CVD and six persons with diabetes. This left 267 individuals (49\% women) without major chronic diseases and with complete data on exposure, outcomes and potential confounders to be included in the analyses.

Characteristics of the study participants are shown in Table 1. The median intake of R-TFA was $1.3 \mathrm{~g} / \mathrm{d}$ ( 5 th percentile (P5), 95th percentile (P95): $0.4,2.7 \mathrm{~g} / \mathrm{d}$ ) or $0.6 \%$ of total energy intake (P5, P95: 0.2, 1.1\%) among all participants. Histograms of the distribution of R-TFA intake are shown in Supplementary Fig. 1 and information on dietary and lifestyle factors by quintiles of R-TFA intake is provided in Supplemental Table 1 (see online supplementary material). The average annual change in weight was $0.4 \mathrm{~kg}$ (P5, P95: $-0.8,1.8 \mathrm{~kg}$ ), whereas the average annual changes in waist and hip circumference were

Table 1 Characteristics of the study population, men and women aged 35-65 years from the Danish MONICA cohort (median values with P5 and P95)

\begin{tabular}{|c|c|c|c|c|c|c|}
\hline & \multicolumn{2}{|c|}{ All participants ( $n$ 267) } & \multicolumn{2}{|c|}{ Men ( $n$ 137) } & \multicolumn{2}{|c|}{ Women $(n 130)$} \\
\hline & Median & P5, P95 & Median & P5, P95 & Median & P5, P95 \\
\hline Age $>50$ years $(\%)$ & \multicolumn{2}{|c|}{45} & \multicolumn{2}{|c|}{40} & \multicolumn{2}{|c|}{50} \\
\hline Weight at baseline $(\mathrm{kg})$ & $70 \cdot 7$ & $52 \cdot 0,93 \cdot 6$ & 75.9 & $61 \cdot 6,95.0$ & 63.3 & $49 \cdot 6,83 \cdot 3$ \\
\hline Annual weight change $(\mathrm{kg})$ & 0.4 & $-0.8,1.8$ & 0.3 & $-0.8,1.9$ & 0.4 & $-0.8,1.8$ \\
\hline Waist circumference at baseline $(\mathrm{cm})$ & 83.5 & $67.5,102.5$ & 88.0 & $75 \cdot 0,105 \cdot 5$ & $76 \cdot 0$ & $65 \cdot 5,96 \cdot 0$ \\
\hline Annual change in waist circumference $(\mathrm{cm})$ & 0.5 & $-1 \cdot 2,2 \cdot 1$ & 0.5 & $-0.9,1.9$ & 0.5 & $-1 \cdot 3,2 \cdot 3$ \\
\hline Hip circumference at baseline $(\mathrm{cm})$ & $97 \cdot 0$ & $88.0,109.5$ & $97 \cdot 0$ & $88.5,107 \cdot 0$ & $97 \cdot 0$ & $87 \cdot 0,111 \cdot 0$ \\
\hline Annual change in hip circumference $(\mathrm{cm})$ & 0.2 & $-0 \cdot 7,1 \cdot 4$ & 0.1 & $-0.6,1 \cdot 1$ & 0.2 & $-0.9,1.6$ \\
\hline Body fat at baseline (\%) & $26 \cdot 3$ & $14 \cdot 1,39 \cdot 4$ & $22 \cdot 6$ & $11 \cdot 9,32 \cdot 0$ & 31.6 & $19 \cdot 0,42 \cdot 9$ \\
\hline Annual change in body fat ( $\%$ point) & 0.4 & $-0.4,1.3$ & 0.3 & $-0.3,1 \cdot 2$ & 0.5 & $-0.6,1.4$ \\
\hline Height $(m)$ & $1 \cdot 70$ & $1.55,1.84$ & 1.77 & $1.63,1.89$ & 1.63 & $1.54,1.74$ \\
\hline BMI at baseline $\left(\mathrm{kg} / \mathrm{m}^{2}\right)$ & $24 \cdot 2$ & $19 \cdot 4,31 \cdot 1$ & 24.4 & $20 \cdot 5,31 \cdot 1$ & 23.9 & $19 \cdot 2,31 \cdot 1$ \\
\hline \multicolumn{7}{|l|}{ Leisure-time physical activity (\%) } \\
\hline Mostly sedentary & \multicolumn{2}{|c|}{21} & \multicolumn{2}{|c|}{21} & \multicolumn{2}{|c|}{20} \\
\hline Light physical activity (>4 h/week) & \multicolumn{2}{|c|}{58} & \multicolumn{2}{|c|}{50} & \multicolumn{2}{|c|}{67} \\
\hline Participating in sports ( $>3 \mathrm{~h} /$ week) & \multicolumn{2}{|c|}{21} & \multicolumn{2}{|c|}{29} & \multicolumn{2}{|c|}{13} \\
\hline \multicolumn{7}{|l|}{ Smoking (\%) } \\
\hline No or occasional & \multirow{2}{*}{\multicolumn{2}{|c|}{$\begin{array}{l}60 \\
40\end{array}$}} & \multicolumn{2}{|c|}{56} & \multicolumn{2}{|c|}{63} \\
\hline Daily & & & \multicolumn{2}{|c|}{44} & \multicolumn{2}{|c|}{37} \\
\hline \multicolumn{5}{|l|}{ Education (\%) } & & \\
\hline$\leq 7$ years & \multirow{2}{*}{\multicolumn{2}{|c|}{$\begin{array}{l}22 \\
47\end{array}$}} & \multicolumn{2}{|c|}{18} & \multicolumn{2}{|c|}{25} \\
\hline $8-11$ years & & & \multirow{2}{*}{\multicolumn{2}{|c|}{$\begin{array}{l}43 \\
39\end{array}$}} & & \\
\hline$\geq 12$ years & \multicolumn{2}{|c|}{31} & & & & \\
\hline Energy intake (MJ/d) & 8.4 & $5 \cdot 1,14 \cdot 0$ & $10 \cdot 2$ & $6 \cdot 5,15 \cdot 3$ & $7 \cdot 2$ & $4.8,10 \cdot 0$ \\
\hline Intake of R-TFA & & & & & & \\
\hline $\mathrm{g} / \mathrm{d}$ & 1.3 & $0.4,2.7$ & 1.4 & $0.4,2.9$ & 1.2 & $0.3,2.4$ \\
\hline E\% & 0.6 & $0.2,1 \cdot 1$ & 0.5 & $0.2,1 \cdot 1$ & 0.7 & $0.2,1 \cdot 1$ \\
\hline Weighted intake of foods high in I-TFA (g/d) & 188 & 28,385 & 215 & 35,452 & 154 & 26,288 \\
\hline $\mathrm{Ca}$ intake $(\mathrm{mg} / \mathrm{d})$ & 850 & 400,1659 & 965 & 364,1835 & 819 & 417,1465 \\
\hline Protein & & & & & & \\
\hline$g / d$ & 71.2 & $46 \cdot 7,117 \cdot 0$ & $79 \cdot 3$ & $52 \cdot 8,124 \cdot 2$ & 62.4 & $43 \cdot 2,88 \cdot 4$ \\
\hline $\mathrm{E} \%$ & 14.3 & $11 \cdot 2,19 \cdot 3$ & 13.8 & $10 \cdot 8,18 \cdot 2$ & $15 \cdot 4$ & $11.4,19.9$ \\
\hline
\end{tabular}

MONICA, MONItoring of trends and determinants in CArdiovascular diseases; P5, 5th percentile; P95, 95th percentile; R-TFA, ruminant trans-fatty acids; $\mathrm{E} \%$, percentage of energy intake; I-TFA, industrially produced trans-fatty acids. 
$0.5 \mathrm{~cm}(\mathrm{P} 5, \mathrm{P} 95:-1 \cdot 2,2 \cdot 1 \mathrm{~cm})$ and $0.2 \mathrm{~cm}(\mathrm{P} 5, \mathrm{P} 95:-0.7$, $1.4 \mathrm{~cm}$ ), respectively. During follow-up the median body fat percentage changed from $26 \cdot 3$ to $28 \cdot 3 \%$, equivalent to an average annual change in body fat of 0.4 percentage points. In absolute terms, the median gain in body fat was $0.4 \mathrm{~kg}$ (P5, P95 percentile: $-0.4,1.6 \mathrm{~kg}$ ) per year.

There were no significant associations between intake of R-TFA and average annual changes in body weight or waist and hip circumference (Figs 1, 2 and 3). Similarly, no significant association was observed between consumption of R-TFA and change in fatness (Fig. 4). None of the associations were statistically significantly non-linear and linear association estimates are provided in Table 2. No significant interaction with sex was found. The correlation between R-TFA and Ca intake was $r=0.5$ as was the correlation between R-TFA and protein intake, but additional adjustment for $\mathrm{Ca}$ and protein intake did not change the results (Supplementary Figs 2-5 and Supplementary Table 2, online supplementary material).

\section{Discussion}

In the present study, intake of R-TFA, assessed by a diet history interview, was not associated with subsequent changes in anthropometry (body weight, waist and hip circumference), measured by trained technicians, or body composition (body fat percentage) obtained by bioelectrical impedance analysis. The range of the $95 \%$ confidence intervals suggests that any relevant associations between intake of R-TFA and changes in these anthropometric measures are unlikely to have produced the available observations.

The participation was relatively high; $79 \%$ of those invited gave a diet history interview at baseline and $76 \%$ of these also participated in the follow-up examination.
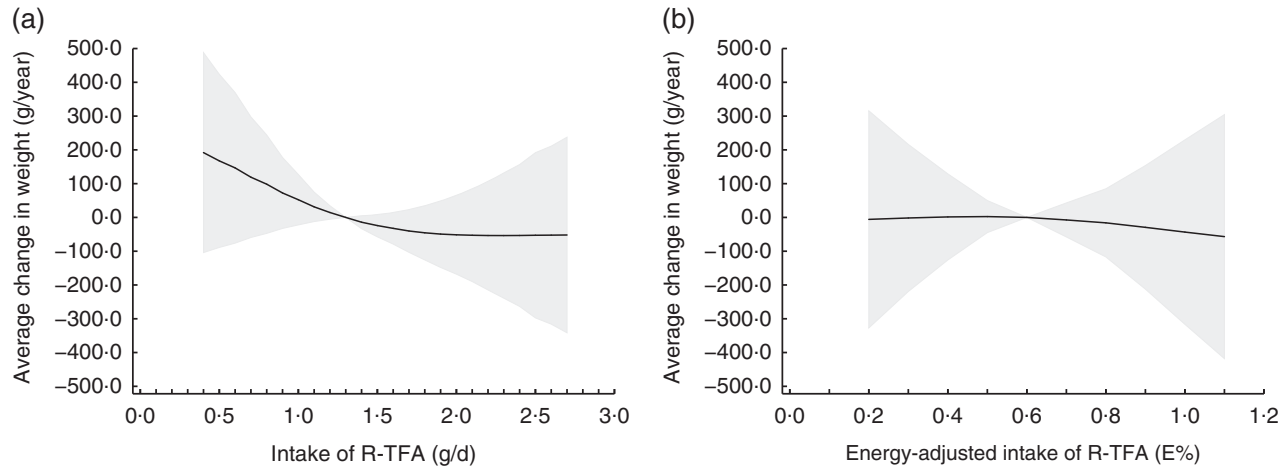

Fig. 1 Absolute (a) and energy-adjusted (b) intake of R-TFA and changes in body weight among 267 men and women aged 35-65 years from the Danish MONICA cohort. Solid lines represent intake of R-TFA modelled with restricted cubic splines (three knots) and shaded areas represent the $95 \%$ confidence interval. Adjustments made for sex, age, baseline BMI, changes in smoking habits, education, intake of foods containing high amounts of I-TFA, and in the analysis of E\% from R-TFA (b), also leisure-time physical activity and total energy intake. $P$ values from test of association: absolute intake $(\mathrm{g} / \mathrm{d})=0.35 ; \mathrm{E} \%$ from R-TFA $=0.95$ (R-TFA, ruminant trans-fatty acids; MONICA, MONItoring of trends and determinants in CArdiovascular diseases; I-TFA, industrially produced trans-fatty acids; E\%, percentage of energy intake)
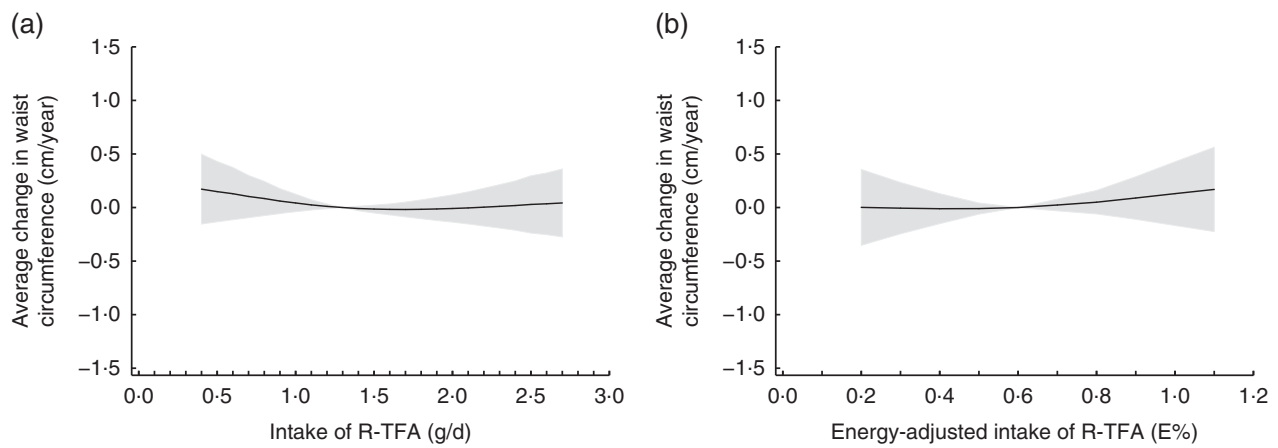

Fig. 2 Absolute (a) and energy-adjusted (b) intake of R-TFA and changes in waist circumference among 267 men and women aged 35-65 years from the Danish MONICA cohort. Solid lines represent intake of R-TFA modelled with restricted cubic splines (three knots) and shaded areas represent the $95 \%$ confidence interval. Adjustments made for sex, age, baseline BMI, baseline waist circumference, changes in smoking habits, education, intake of foods containing high amounts of I-TFA, and in the analysis of E\% from R-TFA (b), also leisure-time physical activity and total energy intake. $P$ values from test of association: absolute intake $(\mathrm{g} / \mathrm{d})=0.58 ; \mathrm{E} \%$ from R-TFA $=0.67$ (R-TFA, ruminant trans-fatty acids; MONICA, MONItoring of trends and determinants in CArdiovascular diseases; I-TFA, industrially produced trans-fatty acids; E\%, percentage of energy intake) 
(a)

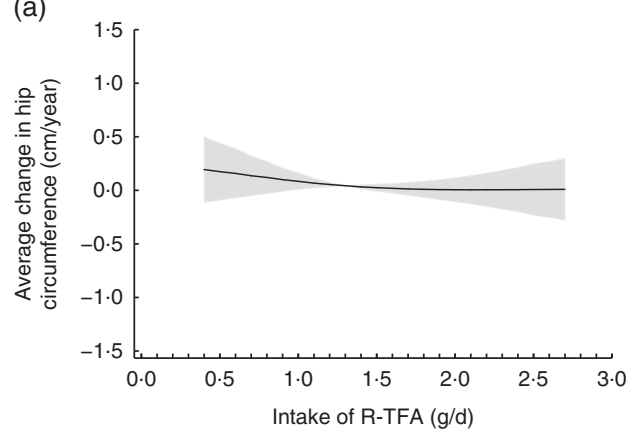

(b)

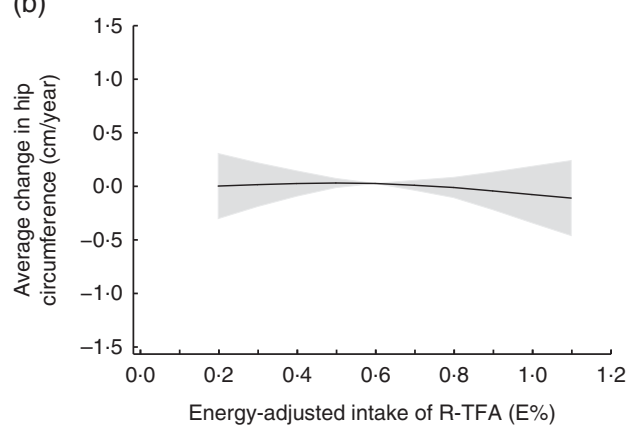

Fig. 3 Absolute (a) and energy-adjusted (b) intake of R-TFA and changes in hip circumference among 267 men and women aged 35-65 years from the Danish MONICA cohort. Solid lines represent intake of R-TFA modelled with restricted cubic splines (three knots) and shaded areas represent the $95 \%$ confidence interval. Adjustments made for sex, age, baseline BMI, baseline hip circumference, changes in smoking habits, education, intake of foods containing high amounts of I-TFA, and in the analysis of E\% from R-TFA (b), also leisure-time physical activity and total energy intake. $P$ values from test of association: absolute intake $(\mathrm{g} / \mathrm{d})=0.52$; $\mathrm{E} \%$ from R-TFA $=0.74$ (R-TFA, ruminant trans-fatty acids; MONICA, MONItoring of trends and determinants in CArdiovascular diseases; I-TFA, industrially produced trans-fatty acids; E\%, percentage of energy intake)
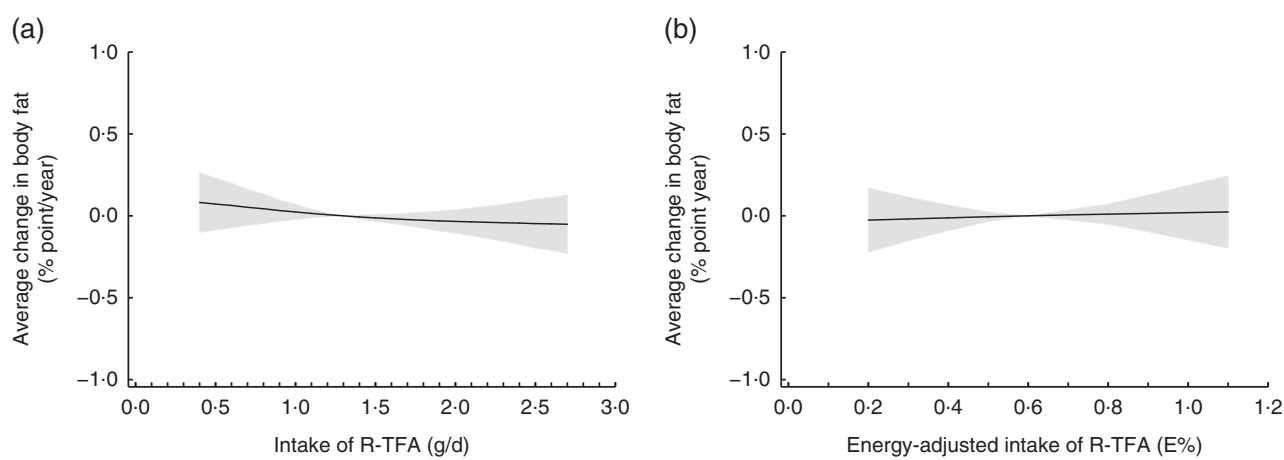

Fig. 4 Absolute (a) and energy-adjusted (b) intake of R-TFA and changes in fat mass (body fat percentage) among 267 men and women aged 35-65 years from the Danish MONICA cohort. Solid lines represent intake of R-TFA modelled with restricted cubic splines (three knots) and shaded areas represent the $95 \%$ confidence interval. Adjustments made for sex, age, baseline BMI, baseline body fat mass, changes in smoking habits, education, intake of foods containing high amounts of I-TFA, and in the analysis of $\mathrm{E} \%$ from R-TFA (b), also leisure-time physical activity and total energy intake. $P$ values from test of association: absolute intake $(\mathrm{g} / \mathrm{d})=0.48 ; \mathrm{E} \%$ from R-TFA $=0.92$ (R-TFA, ruminant trans-fatty acids; MONICA, MONItoring of trends and determinants in CArdiovascular diseases; I-TFA, industrially produced trans-fatty acids; E\%, percentage of energy intake)

Table 2 Linear regression coefficients for the associations between intake of R-TFA and average annual changes in body weight, waist circumference, hip circumference and body fat among 267 men and women aged 35-65 years from the Danish MONICA cohort

\begin{tabular}{lccrr}
\hline & \multicolumn{2}{c}{ R-TFA (g/d) } & \multicolumn{2}{c}{ R-TFA (E\%) } \\
\cline { 2 - 5 } & $\beta$ & $95 \% \mathrm{Cl}$ & \multicolumn{2}{c}{$\beta$} \\
\hline Weight (kg/year) & -0.095 & $-0.249,0.058$ & -0.056 & $-0.507,0.395$ \\
Waist circumference (cm/year) & -0.04 & $-0.21,0.13$ & 0.19 & $-0.30,0.68$ \\
Hip circumference (cm/year) & -0.07 & $-0.22,0.08$ & -0.12 & $-0.53,0.28$ \\
Body fat (\% point/year) & -0.05 & $-0.15,0.04$ & 0.06 & $-0.22,0.33$ \\
\hline
\end{tabular}

R-TFA, ruminant trans-fatty acids; MONICA, MONItoring of trends and determinants in CArdiovascular diseases; E\%, percentage of energy intake; I-TFA, industrially produced trans-fatty acids.

Adjustments made for sex, age, baseline BMI, baseline waist circumference (only in the analyses of change in waist circumference), baseline hip circumference (only in the analyses of change in hip circumference), baseline body fat percentage (only in the analyses of change in body fat), changes in smoking habits, education, weighted intake of foods containing high amounts of I-TFA, and in the analyses of E\% from R-TFA, also leisure-time physical activity and total energy intake.

Loss of participants before baseline may compromise the generalisation of the study result, but is assumed not to cause selection bias. Loss of participants during follow-up, however, could potentially introduce selection bias. When comparing those who did not participate at follow-up with those who did, non-participants on average had higher anthropometric measures and body fat percentages and lower intakes of R-TFA at baseline, but the differences 
were small and not statistically significant. Thus, selection bias is probably of limited concern.

Data on intake of R-TFA were collected using diet history interview at baseline, which may reflect the habitual diet of the participants. Also, the diet history interview may be less sensitive to under-reporting of energy intake as compared with $24 \mathrm{~h}$ recalls and food records ${ }^{(31)}$. However, the participants may have changed their diet during follow-up. In addition, measurement errors are inevitable when participants are asked to recall previous dietary intake. If this misreporting is random, it will generally lead to an underestimation of the true association. A considerable strength of the study is that all anthropometric measures as well as the bioelectrical impedance measurements were obtained under standardised conditions by trained technicians. Furthermore, the technicians were unaware of the participants' dietary exposure to TFA. Information bias is therefore unlikely to have affected the results.

A limitation of the present study is the relatively small study size, which might reduce the power to detect an association between TFA and adiposity. This being said, the anthropometric measurements were obtained by trained technicians and thus less affected by measurement error compared with larger cohort studies, which have relied on self-reported anthropometric measures. Less measurement error would increase the power to detect a possible association. Also, the $95 \%$ confidence intervals are generally narrow and thus any relevant associations between intake of R-TFA and changes in the anthropometric measures are unlikely to have produced the available observations.

Some studies have suggested that $\mathrm{Ca}$ intake protects against weight gain ${ }^{(25,32)}$. The main dietary source of both R-TFA and $\mathrm{Ca}$ is dairy products, and it follows that adjustment for $\mathrm{Ca}$ intake changes the interpretation of the results, as variation in R-TFA is then restricted to food sources not contributing to variation in Ca intake. Adjustment for $\mathrm{Ca}$ and protein intakes in the supplemental analyses did not change the results; however, confounding from other components in dairy products or risk factors not taken into account cannot be excluded.

Whether adjustment for total energy intake is appropriate depends on the mechanisms behind the association between TFA intake and changes in anthropometry ${ }^{(33)}$. If TFA are metabolised in proportion to total energy intake, R-TFA intake adjusted for total energy intake may be most biologically relevant ${ }^{(33)}$. On the other hand, if R-TFA affect satiety and energy intake, adjusting for energy intake would be controlling for an intermediate variable on the causal pathway between R-TFA intake and anthropometric changes. At present, we have limited insight into the mechanisms behind an association between TFA and adiposity, and therefore the optimal approach to attain further insight may be to study both the absolute and the energy-adjusted intake of R-TFA. In the analysis of weight change, a tendency to an inverse association at intake levels up to $1.5 \mathrm{~g} / \mathrm{d}$ was observed, whereas no association was observed when investigating percentage of energy intake from R-TFA. No differences were observed between the analyses of absolute and energy-adjusted intake for the other anthropometric measures and body fat percentage.

The association between intake of R-TFA and changes in anthropometry and body composition may be modified by the amount of fat mass at baseline, but the rather small sample size of the present study excluded the possibility to investigate effect modification using stratified analyses. Yet, former studies have suggested that BMI may modify the association between TFA and weight change ${ }^{(13,14)}$.

We found no associations between R-TFA intake and subsequent changes in body weight and waist circumference in the present study, which is in agreement with previous studies of R-TFA ${ }^{(14-16)}$. In contrast, positive associations between total intake of TFA and changes in body weight and waist circumference were reported in the Nurses' Health Study and the Health Professionals' FollowUp Study ${ }^{(12,13)}$. The TFA intake estimated in these studies may primarily stem from industrial sources as R-TFA generally accounts for approximately $20 \%$ of the total TFA intake in the USA ${ }^{(10)}$. TFA from both industrial and ruminant sources mainly contain trans isomers of octadecenoic acid (18:1), but the distribution of isomers differs between the two sources ${ }^{(34)}$. In industrial partially hydrogenated oils, elaidic acid (trans 9-18:1 $(18: 1 \Delta 9 t)$ ) is the most abundant trans 18:1 isomer, but trans 10-18:1 (18:1 $\Delta 10 t)$ is also present in relatively large amounts. In contrast, vaccenic acid is typically the most abundant trans 18:1 isomer in ruminant fat, although 18:1 $\Delta 10 t$ may replace vaccenic acid in ruminants fed high-concentrate diets ${ }^{(35)}$. In addition, rumenic acid, an isomer of CLA, is present in ruminant fat. A crosssectional study of the proportion of individual TFA isomers in adipose tissue and anthropometric measures suggested that the different TFA isomers may have divergent effects on adiposity ${ }^{(17)}$. However, this was not confirmed in a follow-up study reporting no associations between total adipose tissue TFA or individual adipose tissue TFA isomers and changes in weight and waist circumference ${ }^{(36)}$.

A steady weight state may be reached after a change in diet. This could explain why no associations were observed between habitual intake of R-TFA and subsequent changes in anthropometry and body fat percentage in our study. Future studies of R-TFA and adiposity should preferably look into changes in diet in relation to subsequent changes in anthropometry. This would require measurements from at least three time points, which is actually available in MONICA. However, the study size would be reduced to only 136 persons and therefore it was decided not to conduct these analyses in the present study.

\section{Conclusion}

In conclusion, intake of R-TFA, assessed by diet history interview, was not statistically significantly associated with 
subsequent changes in anthropometric measures of body size and shape (body weight, waist and hip circumference) or body composition (body fat percentage) and the range of the $95 \%$ confidence intervals suggests that any relevant associations are unlikely to have produced the available observations. Thus, the present study supports the findings of previous studies of R-TFA intake and body weight and waist circumference ${ }^{(14-16)}$ and provides evidence that the natural occurrence of TFA in dairy and meat products from ruminants is an issue of no concern to public health.

\section{Acknowledgements}

Financial support: This work is part of the project 'Health Promoting Effect of Milk-Derived Products', which is supported by the Danish Council for Strategic Research (Contract 2101-08-0053) and the Danish Dairy Research Foundation. The funders had no role in the design, analysis or writing of this article. Conflict of interest: The authors declare no conflict of interest. Authorship: C.P.H., K.O. and M.U.J. formulated the research questions and designed the study; B.L.H. collected the data; C.P.H. analysed the data and drafted the manuscript; C.P.H., B.L.H., T.I.A.S., K.O. and M.U.J. contributed to interpretation of the data and critical revision of the manuscript. Ethics of human subject participation: This study was conducted according to the guidelines laid down in the Declaration of Helsinki and all procedures involving human subjects were approved by the ethics committee for Copenhagen County. Written informed consent was obtained from all participants.

\section{Supplementary material}

To view supplementary material for this article, please visit http://dx.doi.org/10.1017/S1368980015001858

\section{References}

1. Turpeinen AM, Mutanen M, Aro A et al. (2002) Bioconversion of vaccenic acid to conjugated linoleic acid in humans. Am J Clin Nutr 76, 504-510.

2. Haug A, Hostmark AT \& Harstad OM (2007) Bovine milk in human nutrition - a review. Lipids Health Dis 6, 25.

3. Gebauer SK, Psota TL \& Kris-Etherton PM (2007) The diversity of health effects of individual trans fatty acid isomers. Lipids 42, 787-799.

4. Wang Y \& Jones PJ (2004) Dietary conjugated linoleic acid and body composition. Am J Clin Nutr 79, 6 Suppl., 1153S-1158S.

5. Leth T, Jensen HG, Mikkelsen AA et al. (2006) The effect of the regulation on trans fatty acid content in Danish food. Atheroscler Suppl 7, 53-56.

6. Stender S \& Dyerberg J (2004) Denmark is the first county in the world to forbid the use of industrially produced fatty acids. Ugeskr Laeger 166, 29-32.

7. Jakobsen MU, Bysted A, Andersen NL et al. (2006) Intake of ruminant trans fatty acids in the Danish population aged 1-80 years. Eur J Clin Nutr 60, 312-318.
8. Doell D, Folmer D, Lee H et al. (2012) Updated estimate of trans fat intake by the US population. Food Addit Contam Part A Chem Anal Control Expo Risk Assess 29, 861-874.

9. Craig-Schmidt MC (2006) World-wide consumption of trans fatty acids. Atheroscler Suppl 7, 1-4.

10. Huth PJ (2007) Do ruminant trans fatty acids impact coronary heart disease risk? Lipid Technol 19, 59-62.

11. Thompson AK, Minihane AM \& Williams CM (2011) Trans fatty acids and weight gain. Int J Obes (Lond) $\mathbf{3 5}$, 315-324.

12. Koh-Banerjee P, Chu NF, Spiegelman D et al. (2003) Prospective study of the association of changes in dietary intake, physical activity, alcohol consumption, and smoking with 9-y gain in waist circumference among 16587 US men. Am J Clin Nutr 78, 719-727.

13. Field AE, Willett WC, Lissner L et al. (2007) Dietary fat and weight gain among women in the Nurses' Health Study. Obesity (Silver Spring) 15, 967-976.

14. Hansen CP, Berentzen TL, Halkjaer J et al. (2012) Intake of ruminant trans fatty acids and changes in body weight and waist circumference. Eur J Clin Nutr 66, 1104-1109.

15. Yamada M, Sasaki S, Murakami K et al. (2009) Association of trans fatty acid intake with metabolic risk factors among free-living young Japanese women. Asia Pac J Clin Nutr 18, 359-371.

16. Nielsen BM, Nielsen MM, Jakobsen MU et al. (2011) A crosssectional study on trans-fatty acids and risk markers of CHD among middle-aged men representing a broad range of BMI. Br J Nutr 106, 1245-1252.

17. Smit LA, Willett WC \& Campos H (2010) Trans-fatty acid isomers in adipose tissue have divergent associations with adiposity in humans. Lipids 45, 693-700.

18. Møller A (1986) Food Composition Tables 1985. Søborg: National Food Agency (in Danish).

19. Leth T, Ovesen L \& Hansen K (1998) Fatty acid composition of meat from ruminants, with special emphasis on trans fatty acids. JAOCS $\mathbf{7 5}, 1001-1005$.

20. Leth T, Bysted A, Hansen K et al. (2003) Trans FA content in Danish margarines and shortenings. JAOCS 80, 475-478.

21. Heitmann BL (1990) Prediction of body water and fat in adult Danes from measurement of electrical impedance. A validation study. Int J Obes 14, 789-802.

22. Saltin B \& Grimby G (1968) Physiological analysis of middle-aged and old former athletes. Comparison with still active athletes of the same ages. Circulation 38, 1104-1115.

23. Willett WC, Howe GR \& Kushi LH (1997) Adjustment for total energy intake in epidemiologic studies. Am J Clin Nutr 65, 4 Suppl., 1220S-1228S.

24. Zemel MB (2003) Role of dietary calcium and dairy products in modulating adiposity. Lipids 38, 139-146.

25. Barba G \& Russo P (2006) Dairy foods, dietary calcium and obesity: a short review of the evidence. Nutr Metab Cardiovasc Dis 16, 445-451.

26. Christensen R, Lorenzen JK, Svith CR et al. (2009) Effect of calcium from dairy and dietary supplements on faecal fat excretion: a meta-analysis of randomized controlled trials. Obes Rev 10, 475-486.

27. Westerterp-Plantenga MS, Lemmens SG \& Westerterp KR (2012) Dietary protein - its role in satiety, energetics, weight loss and health. Br J Nutr 108, Suppl. 2, S105-S112.

28. Santesso N, Akl EA, Bianchi M et al. (2012) Effects of higherversus lower-protein diets on health outcomes: a systematic review and meta-analysis. Eur J Clin Nutr 66, 780-788.

29. Harrell FE (2001) Regression Modeling Strategies. With Applications to Linear Models, Logistic Regression and Survival Analysis, vol. 1. New York: Springer.

30. Greenland S (1995) Dose-response and trend analysis in epidemiology: alternatives to categorical analysis. Epidemiology 6, 356-365. 
31. Black AE, Goldberg GR, Jebb SA et al. (1991) Critical evaluation of energy intake data using fundamental principles of energy physiology: 2. Evaluating the results of published surveys. Eur J Clin Nutr 45, 583-599.

32. Teegarden D \& Zemel MB (2003) Dairy product components and weight regulation: symposium overview. J Nutr 133, issue $1,2435-244 S$.

33. Willett W (1998) Nutritional Epidemiology, 2nd ed. New York: Oxford University Press.
34. Stender S, Astrup A \& Dyerberg J (2008) Ruminant and industrially produced trans fatty acids: health aspects. Food Nutr Res 2008, 52.

35. Shingfield KJ, Bonnet M \& Scollan ND (2013) Recent developments in altering the fatty acid composition of ruminant-derived foods. Animal 7, Suppl. 1, 132-162.

36. Hansen CP, Berentzen TL, Ostergaard JN et al. (2014) Adipose tissue trans-fatty acids and changes in body weight and waist circumference. Br J Nutr 111, 1283-1291. 